\title{
Évaluer une pratique innovante : essai réflexif autour de l'expression théâtrale
}

\section{Marie-Noëlle Cocton}

\section{(2) OpenEdition}

1 Journals

\section{Electronic version}

URL: http://journals.openedition.org/ripes/2116

DOI: 10.4000/ripes.2116

ISSN: 2076-8427

\section{Publisher}

Association internationale de pédagogie universitaire

\section{Electronic reference}

Marie-Noëlle Cocton, «Évaluer une pratique innovante : essai réflexif autour de l'expression théâtrale », Revue internationale de pédagogie de l'enseignement supérieur [Online], 35(2) | 2019, Online since 13 March 2020, connection on 08 September 2020. URL : http://journals.openedition.org/ripes/2116 DOI : https://doi.org/10.4000/ripes.2116

This text was automatically generated on 8 September 2020

Article L.111-1 du Code de la propriété intellectuelle. 


\title{
Évaluer une pratique innovante : essai réflexif autour de l'expression théâtrale
}

\author{
Marie-Noëlle Cocton
}

\section{Introduction}

1 Innover n'est ni un « gadget » ni une « bouffée d'oxygène » (Cros, 2017, p. 180). Innover consiste à mettre intentionnellement du neuf dans une pratique habituelle antérieure (De Ketele, 2010, p. 8). Encore faut-il que la nouveauté se transmette et qu'elle se diffuse dans la société pour faire évoluer les modes de penser, de parler et de faire!

2 L'innovation est, de toute évidence, à prescrire dans le cahier des charges de formation des enseignants pour devenir une véritable compétence professionnelle (Rouby et Thomas, 2014). C'est ce que nous tentons de faire, depuis quelques années, dans le cadre de la formation de master en Didactique des Langues, parcours Français Langue Étrangère ${ }^{1}$, en proposant une réflexion sur la mise en œuvre d'une pratique innovante, dans le cadre du cours "Pratiques théâtrales et artistiques». La démarche consiste à prouver aux futurs enseignants de FLE que le corps est moteur dans la compétence à communiquer langagièrement. Cette démonstration s'appuie sur l'expérimentation d'une pratique dite «innovante » : la mise en œuvre d'un cours intitulé « expression théâtrale » auprès d'un public d'apprenants étrangers en contexte immersif en France. Cette pratique, au départ intuitive, a permis, par le biais de différentes formes d'évaluation, de remodeler ses objectifs et de procéder à des réajustements. En effet, si l'innovation est un élément intrinsèque de l'enseignement, elle nécessite de pouvoir être évaluée en vue de (se) "métamorphoser». Reste à savoir, dans le cadre de pratiques pédagogiques innovantes, ce qu'«évaluer» veut dire. Et s'il convient de parler d'« innovation» lorsqu'il s'agit, pour l'enseignant-chercheur, d'une pratique d'enseignement-apprentissage implémentée depuis dix ans. Sans oublier de prendre en compte l'institution et surtout, l'apprenant, comme acteur social intégré au processus 
d'enseignement-apprentissage et, par conséquent, à celui du "surgissement d'inédit " (De Ketele, 2002, p. 47) que représente l'innovation pédagogique.

3 Cet article s'inscrit dans le champ de la didactique des langues. Notre approche se veut narrative et réflexive (Jorro, 2002) et entend proposer un regard descriptif dans le but de conscientiser une pratique d'enseignement-apprentissage du français langue étrangère. Pour cela, nous reviendrons tout d'abord sur le concept d'innovation pédagogique et justifierons l'innovation que représente ce cours; puis, nous porterons un regard sur l'évaluation envisagée pour ce cours et ses aspects novateurs; enfin, nous nous intéresserons aux dires de l'apprenant consignés dans l'outil nommé carnet de bord.

\section{Ce qu'innover veut dire}

Innover, nous rappelle Françoise Cros (2013, p.169), est une pratique à connotation positive récente dont la définition "reste floue, voire même doit le rester si [le syntagme] veut garder son pouvoir de persuasion et de dynamique ». Avant les années 1960, le fait d'introduire une nouveauté dans un usage était perçu comme néfaste pour l'évolution de la société. L'innovation, précise Cros (1999), s'est d'abord montrée économique puis technologique avant de devenir processus. Pour être de son époque, il faut faire preuve d'initiative, de créativité dans un environnement en perpétuel changement. En enseignement, une pratique innovante implique un changement qui s'éloigne de la norme et qui augmente la qualité des apprentissages des étudiants : "l'innovation est pédagogique, lorsqu'elle cherche à améliorer substantiellement les apprentissages des étudiants en situation d'interaction et d'interactivité » (Bédard et Béchard, 2009, p. 36). Dans un article de 2010 traitant de l'innovation pédagogique dans l'enseignement supérieur, De Ketele met, lui aussi, en exergue l'innovation en tant que champ complexe et paradoxal multipliant les points d'entrée et ce qu'il nomme les "strates» (De Ketele, 2002, p. 14), entendues comme "moments» (Cros, 1999) ou dynamiques de l'innovation. Selon lui, la première constitue le "primat de l'informel » ou « des chemins de traverses de l'innovation». Il ajoute que «le(ur) point de départ est souvent une difficulté perçue comme insoutenable et une frustration qui amènent ces personnes (les innovateurs) à rechercher des nouvelles façons de faire. Cela résout ainsi le problème identifié et tente de lever le sentiment de frustration " (De Ketele, 2002, p. 15). Cette première strate, généralement abandonnée car soutenue par un innovateur isolé, peut aboutir à une seconde strate «du primat formel » comprise comme l'acceptation institutionnelle et donc, l'intégration d'une innovation a priori personnelle. La troisième strate, commente-t-il, « s'explique par le caractère paradoxal du concept d'innovation : une fois que l'on a introduit du neuf, le neuf devient la norme et n'est plus de l'innovation" (De Ketele, 2002, p. 17). Si l'innovation possède une « temporalité non linéaire » (Cros, 2001, p. 75), composée de moments d'accélération, de ralentissement, ou de moments qui surgissent de façon opportune, elle a pour vocation première de mettre en action et en mouvement, ce qui n'est pas sans rappeler l'étymologie d'une autre notion: la motivation. Ainsi, la source de l'innovation a des origines diverses: difficulté ou insatisfaction personnelles, envie de changement ou intention personnelles, projets d'action ou recommandations, voire injonctions institutionnelles. En visant l'amélioration en permanence, l'institution est, de fait, la première génératrice d'innovation. En témoigne, la mise en place, en avril 2013, du conseil national de l'innovation pour la réussite éducative (CNIRE), prescrit par le 
ministère français de l'Éducation Nationale. Innover, c'est admettre qu'il n'existe pas une, mais des façons de faire. C'est donc porter un regard décentré, faire appel à une conscience ou une intuition professionnelle qui invite à orienter l'action, à imaginer des outils, des stratégies, des gestes pédagogiques adaptés aux finalités que l'enseignant poursuit.

\subsection{Innover en classe : le choix de l'expression théâtrale}

5 En créant le cours d'« expression théâtrale $»^{2}$ en février 2008 au Centre International d'Études Françaises (CIDEF) de l'Université Catholique de l'Ouest à Angers, il était d'abord question de finalité : permettre aux apprenants de français langue étrangère d'inscrire l'acte de communiquer en langue étrangère dans sa dimension holistique, se rapprochant ainsi de la définition de la communication proposée par l'école de Palo Alto, « un processus social permanent intégrant de multiples codes de comportement: la parole, le geste, le regard, la mimique, l'espace interindividuel, etc. » (Winkin, 1981, p. 24). Il était aussi question d'innover, c'est-à-dire de tenter de lever la frustration en s'opposant à une pratique existante: la "production orale », plébiscitée par le Cadre Commun de Référence pour les Langues (CECRL) ou fortement influencée par les certifications de type DELF ${ }^{3}$. À l'époque, il existait un sentiment d'insatisfaction personnelle quant à la façon habituelle de travailler cette compétence : de notre point de vue d'enseignants, il importait de dépasser la notion de "production " pour aller vers l'« expression ", qui, au-delà de la fabrication de sons, de phrases et de mots qui font référence à la langue, englobe le silence, le corps, la gestuelle, les émotions qui sont, eux, des éléments faisant référence au langage; il manquait aux critères d'évaluation traditionnels que peuvent être la maitrise du lexique, du système phonologique ou de la morphosyntaxe, des critères extralinguistiques tels que la spontanéité, l'engagement dans l'action, et la créativité.

6 Cette démarche, au départ personnelle, a rapidement trouvé appui au niveau institutionnel. Petit à petit, la terminologie «expression théâtrale » a été adoptée : il s'agissait d'écarter intentionnellement le "théâtre ", en tant que genre littéraire (même si des textes de théâtre sont souvent utilisés comme supports d'activités); "l'esthétique ", travail passionnant que poursuit Pierra (2006) sur la beauté du texte, la beauté ressentie par l'apprenant-spectateur et surtout celle qu'il dégage quand il se met à habiter le texte; ainsi que le « projet théâtral » tel qu'il est notamment décrit par Payet (2010). L'objectif était et reste l'expression extériorisée - qu'elle soit idée, émotion, pensée, parole, geste, regard, écoute, attention ou disponibilité - sans forcément l'adosser à la "pratique » au sens d'application directe d'une théorie ou de formation à la " pratique » théâtrale pour devenir acteur. Le but est de se centrer à la fois sur l'apprenant, dans son rapport au langage dans le contexte d'apprentissage d'une langue étrangère, et à l'individu, dans son rapport à l'autre, aux autres, à soi. L'ambition est de mener à une expression, quelle qu'elle soit, en favorisant une dimension spontanée et créative. Tout en veillant à coconstruire avec l'autre, de manière imprévisible, notamment à travers l'activité d'improvisation. En acceptant l'imprévisibilité de l'action, l'enseignant sait que l'enjeu de l'innovation réside dans la mobilisation inédite de compétences. Cet effet de nouveauté n'est pas redouté mais assumé de plein gré. L'innovation est à ce prix : l'innovateur se dit prêt à en découdre avec la réalité, il parie sur l'acte à venir, il se tient prêt. Pour cela, il s'appuie sur sa propre expérience en termes de mobilisation inédite de compétences (dans le cas 
présent, une formation théâtrale en tant qu'artiste amateur et une pratique théâtrale au sein d'un groupe de théâtre), sur les activités et réflexions pédagogiques issues de professionnels du théâtre (les pistes d'improvisation et de techniques du mouvement suggérées par Lecoq (1997); la présence physique et la mécanique du corps présentée de Meyerhold (cité dans Baldwin et Mederos Syssoeva, 1999), sur les activités menées conjointement entre enseignants et artistes (voir, par exemple, la collection Les Ateliers de théatre, proposée par l'ANRAT ${ }^{4}$ ), ou encore celles envisagées pour la didactique du FLE pour le travail sur la voix (Vielmas, 1991) ou les activités classées par niveau (Pierré et Treffandier, 2012).

7 L'innovation, dès lors, tient au caractère inédit de la création du cours dans le cadre de l'enseignement-apprentissage du français langue étrangère au CIDEF; du contenu inhabituel du cours dans l'enseignement-apprentissage du français langue étrangère (la notion de didactique de la pratique théâtrale et sa théorisation n'est apparue que récemment dans le cadre du FLE (Alix, Lagorgette et Rollinat-Levasseur, 2013) - même si tous s'accordent à dire qu'elle pré-existait notamment avec Ryngaert (1977) ou Caré (1983); ainsi que de l'évaluation originale prescrite pour ce cours.

\subsection{Innover dans l'évaluation : les conséquences d'une pratique innovante}

Qui dit cours nouveau dit objectifs à fixer et, par conséquent, évaluation à définir. Sans même évoquer la notation qui reste institutionnelle, il convenait de réfléchir aux modalités d'évaluation les plus appropriées pour non seulement rendre compte de la participation et de l'implication des apprenants dans le cours mais aussi de leurs performances.

9 Les recherches ${ }^{5}$ menées autour de la multicanalité de la communication, largement inspirée des travaux de Tabensky (1997) dans son ouvrage Spontanéité et interaction, avaient permis, au fil du temps, de constituer des grilles d'évaluation ${ }^{6}$ construites autour de deux concepts majeurs : la spontanéité et la créativité. Ce faisant, l'intention pédagogique visait à s'éloigner des critères d'évaluation décrétés par le Conseil de l'Europe (2005) dans les certifications. Il s'agissait d'adopter une évaluation dite " alternative ", ne constituant pas une pensée alternative comme meilleure en soi, mais comme une volonté de proposer « des pistes autres, souvent masquées, marginalisées, voire délégitimées par les tendances actuellement dominantes » (Huver et Springer, 2011, p. 208). L'alternance portait sur les critères, outils et modalités d'évaluation choisis, et non sur le format de la grille ou sa pondération. En didactique des langues, l'évolution de la " compétence à communiquer langagièrement " suppose d'intégrer à l'enseignement et donc à l'évaluation, des compétences autres que linguistiques, ainsi que des modalités d'évaluation qui prennent en compte le caractère dynamique d'une production orale de type interactionnel. Dès lors, la notion de compétence ne pouvait se réduire aux compétences cognitives ni même aux savoir-faire : ce qui la caractérise, c'est la mise en jeu d'un répertoire de ressources (connaissances, attitudes, compétences émotionnelles, relationnelles, etc.) que l'apprenant doit savoir classer et organiser pour "construire une combinatoire de multiples ingrédients" (Le Boterf, 2002, p. 20). Parmi ces compétences, figurent la prise de conscience interculturelle, le savoir-être (attitudes, motivations, traits de personnalité, etc.), la conscience de la langue et de la communication et les aptitudes heuristiques (observation, etc.). Cependant, ces compétences restent minorées dans l'évaluation. L'articulation entre les "compétences langagières» et ces "autres compétences" n'est d'ordinaire pas 
abordée et peu d'éléments sont fournis pour favoriser leur prise en compte et leur valorisation. Pourtant, en observant et en analysant la construction d'un jeu, bien des éléments apparaissaient : l'engagement de l'apprenant dans le jeu, sa spontanéité, sa capacité d'écoute et sa volonté de coopérer. Autant de critères qui renvoient à des compétences dites «sociales" (Morlaix, 2015) non intégrées dans une évaluation exclusivement centrée sur des descripteurs généralement imposés et non modifiables, puisqu'ils correspondent à des standards définis globalement, valables pour tous et dans tous les contextes. Parmi ces compétences sociales, l'on peut distinguer les compétences interindividuelles (comportements vis-à-vis des autres) et intra individuelles (comportements vis-à-vis de soi).

Dans le cadre de notre étude, donc d'un travail collaboratif (celui de la construction d'une expression théâtrale à plusieurs), l'interaction ne pouvait se limiter à des dimensions externes à l'énoncé (tours de parole, séquentialité, etc.), celles-ci se jouant à travers et dans les relations qui s'expriment au sein de l'énoncé et qui sont dépendantes des relations internes. De ce point de vue, l'hypothèse selon laquelle une action ne peut être que meilleure par la volonté intérieure d'un individu, sa disposition à exécuter des mouvements, sa libre spontanéité (sua sponte = de sa propre volonté), avait été formulée (Cocton, 2007). Or, la grille de Tabensky (1997) mettait en évidence que la spontanéité avait un lien avec l'implication dans le jeu ou l'engagement ${ }^{7}$ qui luimême donnait toute sa force à l'interaction et que cette altération jouait sur l'apprentissage. Aussi avions-nous décidé que l'évaluation réservée à l'enseignant prendrait en compte trois profils : celui du joueur, de l'acteur et de l'apprenant. Dans une première partie axée sur le jeu, nous cherchions, à travers la dimension multicanale de la communication, à faire ressortir l'engagement du joueur dans le jeu (prise d'initiative, prise en compte du dire d'autrui), sa spontanéité (réponses immédiates, cohérence face à l'inattendu, capacité à alimenter le scénario) et sa créativité (construction du rôle, adaptation du rôle dans le jeu). Dans une seconde partie axée sur l'apprentissage, nous nous intéressions à la mobilisation de connaissances et à l'utilisation de ces mêmes connaissances (soit à la compétence et à la performance) d'un point de vue langagier. La dernière partie de l'évaluation avait attrait au « spectacle ». La visée de cet article n'est pas de revenir sur l'utilisation de ces grilles dont d'autres articles ont déjà fait mention (Cocton, 2015) mais de nous intéresser au cheminement du praticien, car, rappelons-le, nous situons cet article dans une analyse réflexive du praticien sur sa propre démarche et, de fait, nous tentons de définir ce qu'est l'évaluation d'une pratique innovante.

\section{Ce qu'évaluer une pratique innovante veut dire}

11 Qui dit innovation, dit évaluation; une pratique en engageant une autre. Or, cette pratique diffère selon qui évalue : l'institution cherche à évaluer le cours, son intérêt et son fonctionnement; les membres de l'institution souhaitent être informés pour mieux comprendre l'origine et la mise en œuvre de cette innovation; l'innovateur cherche à améliorer son innovation et vise une satisfaction (autosatisfaction également); enfin, ceux qui vivent l'innovation ne sont pas forcément conscients qu'il s'agit d'une innovation, sauf si elle leur a été décrite comme telle ou qu'elle est, pour eux, une pratique nouvelle. 


\subsection{Point de vue « institutionnel»}

12 Après dix années de mise en œuvre d'une pratique pédagogique, il convient de souligner l'effacement du caractère « inédit » pour au moins deux des pôles du triangle des acteurs de l'innovation: l'enseignant-innovateur et l'institution. Au niveau institutionnel, il n'a jamais été envisagé d'évaluer le caractère innovant du cours mais bien plutôt d'évaluer le cours. À chaque fin de semestre, un formulaire d'évaluation ${ }^{8} \mathrm{du}$ cours est distribué aux apprenants. Cette évaluation a une valeur indicative pour l'institution et pour l'enseignant, notamment en termes de « satisfaction générale » du cours et de la cohérence entre les besoins et les attentes. Cette évaluation permet de valider le maintien du cours d'un semestre à l'autre. Suite aux évaluations, l'institution a continué à valoriser ce cours dans ses programmes. De 2012 à 2018, 285 étudiants ont participé à ce cours, soit une moyenne de 11 apprenants par niveau et par semestre (le maximum étant fixé à 12).

\subsection{Point de vue « enseignants, membres de l'institution »}

13 Au niveau de l'équipe enseignante, il est à noter que l'approche innovante a, au départ, intrigué - «mais ça consiste en quoi, exactement, ton truc ?» (Françoise), « pourquoi est-ce que ta classe fait autant de bruit?» (Cécile), « c'est comme de l'expression orale, non?» (Claudine) - jusqu'à ce que les enseignants soient invités à observer le cours ou à l'expérimenter, en formation, lors de journées pédagogiques. Nous partîmes donc seuls mais, par un prompt renfort, nous nous vîmes cinq à pouvoir/vouloir enseigner ce cours aujourd'hui. C'est parce que l'équipe se construit peu à peu que nous ne cessons de redessiner le cours. Ainsi, au semestre de printemps 2019, nous allons de nouveau innover en proposant un cours à double-voix: d'un côté, une enseignante en phonétique et de l'autre, une enseignante d'expression théatrale. Est attendue la rencontre entre la voix et le corps, ce qui oblige à redéfinir les objectifs, l'évaluation, le contenu des activités et le rythme des séances.

\subsection{Point de vue « innovateur »}

14 Pour évaluer la pratique innovante qu'est l'expression théâtrale, nous nous appuierons sur deux propos : celui de Jorro (2002, p. 22) qui rappelle l'importance « de savoir dire l'action, de savoir la décrire et la questionner ». Cette activité langagière suppose des qualités énonciatives permettant de retracer le fil continu de la pratique et d'en saisir les enjeux, d'en nommer les impasses et les réussites; celui de De Ketele (2002, p. 42) évoquant les cinq critères d'évaluation de l'inédit:

(1) Caractérisation de l'inédit dans la poursuite d'une action;

(2) Mise en œuvre de l'inédit;

(3) Changements proposés par l'enseignant à la suite de cette mise en œuvre;

(4) La case vide;

(5) La réflexivité de l'enseignant.

15 Parmi les changements effectués par l'enseignant suite à la mise en œuvre de cette pratique innovante (critère 3), figurent nombre d'éléments qui ont été améliorés au fil des ans et ce, grâce aux difficultés rencontrées ou suggestions proposées par les apprenants ou collègues-enseignants lors de l'expérimentation: l'introduction progressive des objectifs en début de séance avec une initiation réflexive à ce que 
représente «l'expression »; la prise en compte des commentaires, questions, difficultés ou réactions des apprenants en classe (ex: «comment fait-on pour imaginer?", « comment comprendre la ponctuation?», « comment construire une histoire? », etc.) à travers de nouvelles activités (ex: l'imagination par la fenêtre, la marche de la ponctuation, les techniques du sel et du poivre); la pluralité accordée, au fil des ans, à l'évaluation de l'expression théâtrale avec la mise en place d'une évaluation sommative non-institutionnelle; l'implication des apprenants dans le choix des critères d'évaluation; la participation à la représentation de fin de semestre pour permettre un aboutissement et une réalisation collective (ex: saynètes et improvisations semiguidées lors des soirées dites «internationales»); le visionnage des vidéos d'improvisation avec les apprenants pour une meilleure compréhension des éléments de remédiation; la répétition des scènes pour une remédiation effective; l'apport de la phonétique pour une amélioration de l'articulation et de l'intonation.

\subsection{Point de vue « apprenant »}

16 Au niveau des apprenants, le caractère "inédit » demeure : aujourd'hui, seuls les apprenants peuvent ressentir du «neuf» dans cette pratique puisque d'un cours à l'autre, ce sont de nouveaux apprenants. Rappelons toutefois que « ce qui est neuf pour une personne ou un groupe ne l'est pas nécessairement pour d'autres» (De Ketele, 2002, p. 37). Pour illustrer notre propos, il a semblé pertinent de relire les carnets de bord rédigés par les apprenants. Dans notre démarche pédagogique, le carnet de bord a une dimension d'écriture réflexive, faisant écho ou opposition à la parole spontanée privilégiée en classe. Le carnet de bord constitue un «support d'autoformation qui a une portée à la fois rétroactive et proactive » (Deum, 2004, p. 8) ainsi qu'un outil d'autoévaluation et de développement d'une pensée autonome. Le carnet de bord, en générant une réflexion critique, aide les étudiants «à aller vers une plus grande autonomisation » (Château et Zumbihl, 2010, p. 4), ce qui leur permet de prendre en charge leur apprentissage. Le carnet vise, ainsi, à impliquer l'apprenant dans une démarche réflexive autour de son apprentissage, et à engager une réflexion de l'individu dans la (re)-découverte de son corps et de ses émotions, au contact des autres, en jeu théâtral.

17 L'objectif premier de ce carnet de bord n'était pas de rendre compte de l'innovation de la pratique théâtrale : en effet, au début de chaque semestre, nous posons la question suivante : «Avez-vous déjà suivi un cours d'expression théâtrale ou de théâtre ?» et ce, afin de mieux connaître les connaissances et compétences préalables ainsi que les appréhensions des apprenants. Nous n'avions jamais pensé que le carnet de bord pourrait aussi être un corpus de données intéressant pour évoquer l'innovation'. Mais comme ces carnets sont généralement révélateurs d'une personnalité et d'une intimité profonde, ils servent aussi d'indicateurs de (nouveaux) critères à associer à toute démarche évaluative.

18 Une cinquantaine de carnets de bord a été relue : ils ont été complétés par les étudiants étrangers qui ont choisi le cours d'expression théâtrale entre 2012 et 2016. Le format est laissé à la liberté de chacun : bloc-notes, carnet, cahier, avec ou sans pages blanches, avec ou sans lignes, pour dessiner ou pour écrire, blog, photos ou vidéo pour raconter, à leur manière, ce qu'ils ont vécu au cours du semestre. La consigne est la suivante : « Au fil du semestre, racontez ce que vous vivez, ressentez, comprenez, observez dans ce cours; racontez vos réussites, vos manques, vos besoins, ce qui vous touche, vous 
surprend, vous anime. » Pour ce faire, nous avons utilisé l'analyse de contenus en nous appuyant sur les 5 critères précédemment évoqués (les critères 3 et 5 sont à destination de l'enseignant) :

(1) le caractère « inédit » de la pratique correspondant à " c'est la première fois », « je n'avais jamais fait de théâtre », etc.;

(2) la métamorphose provoquée par une nouvelle pratique;

(4) et la « case vide, c'est-à-dire tout ce qui surgit et qui est complètement à côté de l'action innovante mais ne peut être ignoré » " (De Ketele, 2002, p. 43).

De façon générale, les carnets de bord sont plus descriptifs que réflexifs (à l'inverse des formulaires d'auto-évaluation): les apprenants décrivent les activités effectuées en classe, en précisant s'ils les ont aimées ou non (ce qui est très utile pour l'enseignant qui peut réorienter le contenu du cours si besoin). Ils décrivent certaines improvisations ou jeux de scène en précisant leur admiration pour les autres et par comparaison, leur incapacité à ... De-ci-de-là, ils se laissent aller à l'écriture et livrent des sentiments très personnels.

20 1. Dans l'ensemble, les carnets de bord ne laissent pas de trace d'expérience inédite. Les apprenants ne mentionnent pas le fait qu'ils participent à ce genre de cours pour la première fois, ce qui n'est pas étonnant. Cela vient du fait que nous avons pour habitude de leur poser la question directement en classe, lors de la première séance. Ils sont généralement nombreux à avoir fait du théâtre : certains, au lycée, en vue d'une représentation théâtrale; d'autres, à l'université pour travailler les textes dramaturgiques. La réponse à la deuxième question a, pour le moment, toujours été négative sauf s'ils ont déjà fait un semestre au CIDEF et qu'ils ont choisi ce cours à un niveau inférieur. Il est également peu fait mention d'originalité : « C'est la première fois que je choisis ce cours. Dans la dernière ${ }^{10}$ semestre, puisque j'étais un mois de retard, je ne pouvais pas opter ce cours. Selon mes camarades, c'était un cours intéressant et original. J'étais donc curieuse pour ce cours et l'ai choisi dans cette semestre même si je ne savais rien de l'expression théâtrale." (Weiling, étudiante chinoise). L'inédit concerne également la participation à une courte représentation théâtrale qu'il nous arrive d'effectuer en fin de semestre lors de la soirée internationale ${ }^{11} \mathrm{du}$ CIDEF, mais là encore, peu d'apprenants l'évoquent: «C'est excitant car c'est la première fois que je me retrouve sur une scène: j'espère bien qu'on va créer un spectacle magnifique » (Yao Shen, étudiante chinoise).

21 2. Les métamorphoses ${ }^{12}$ provoquées par la participation et l'implication dans le cours sont multiples. Elles renvoient à la notion de compétences sociales (Morlaix, 2015) et concernent :

22 - la prise de conscience d'un besoin de métamorphose personnelle : «J'ai choisi ce cours parce que je veux devenir plus vive et parler plus naturellement. Maintenant je suis plus confiante à apprendre le français. » (Yao Shen, étudiante chinoise); «Je sais que je n'aime pas ce cours parce que c'est « outside my confort zone » et alors, c'est bien que je sois là, parce que c'est toujours quand on est dehors la zone de confort qu'on apprend le plus » (Samantha, étudiante anglaise); «J'aime cette activité parce qu'elle a forcé moi de parler. Je dois parler plus. » (Hannah, étudiante allemande); «J'ai choisi ce cours parce que je suis timide. Maintenant je n'ai pas peur de parler avec des Français » (Natsuki, étudiante japonaise);

23 - la métamorphose observée tout au long du cours: "Je me sens plus confortable de parler maintenant qu'au début du cours » (Casey, étudiante américaine); 
«Franchement, je n'ai pas appris grand-chose dans la classe d'expression théâtrale comme la grammaire, la phonétique. Pourtant, je suis devenue plus ouverte, plus gaie. » (Yao Shen, étudiante chinoise); "Avant j'ai eu peur de parler devant tout le monde. Mais maintenant, avec ce cours, je peux le faire plus facilement» (Emily, étudiante irlandaise); «Je pense que j'ai gagné beaucoup de confiance grâce à ce cours. J'ai eu aucune difficulté de chanter ou danser devant un public mais comme je n'avais pas beaucoup d'expérience de théâtre, ça m'a aidé, vraiment ! (Eun Kim, étudiant coréen); «Je crois que j'ai progressé grâce à ce cours. Je suis arrivé mieux d'exprimer mes émotions. »(Yuki, étudiante japonaise).

24 - la métamorphose observée concernant une activité précise : «J'ai aimé cette classe car je l'ai trouvée intéressante mais aussi je me sens comme j'ai fait des progrès comme improvisatrice. J’ai observé mon équipe et répondu en conséquence » (Paige, étudiante américaine); «C'était une très bonne activité pour moi parce que je pense trop quand je parle et ça m'a fait parler plus rapide. » (Hannah, étudiante allemande); «Ce qui est difficile pour moi avec le personnage, c'est que je galère à l'imaginer. C'est plus facile pour moi de visualiser l'espace et c'est pour ça que j'ai aimé l'activité » (Rena, étudiante américaine); "Quelque chose que j'ai appris: quand on crée des mini-scènes, en "grommelot", c'était cool de remarquer que quand on ajoute des gestes et des sentiments, l'histoire est normalement claire même si les paroles ne signifient rien. » (Emma, étudiante anglaise); « Aujourd'hui, j'ai gagné un meilleur sens du timing et plus de confiance avec mes paroles. » (Elise, étudiante allemande).

- la métamorphose liée à son rapport à l'autre : «Au début, il était difficile pour moi d'être moins timide. J'ai pensé que tout le monde était en train de me juger quand j'ai fait des choses bizarres. Mais après, j'ai réalisé que personne me juge et je n'ai pas besoin d'être timide. (...) J'ai appris d'être spontanée et faire plus de gestes pour créer la scène » (Emily, étudiante américaine); "Il est étrange de regarder des yeux colorés. C'est la première fois que je l'ai fait » (Mizuki, étudiante japonaise).

26 - la métamorphose liée à son rapport à l'apprentissage : « Je n'avais jamais pensé que la ponctuation avait du mouvement » (Elani, étudiante américaine); « C'est intéressant de savoir que je parle un peu vite quand je lire. Ce n'est pas facile de faire les pauses assez longtemps. En plus, je pense que les silences sont aussi difficiles parce qu'ils portent tellement beaucoup d'informations. Les silences sont très important!!! (je crois).» (Eun Kim, étudiant coréen); "Une des activités marquantes était celle où on a eu une affiche avec des émotions et une personne s'exprimait et les autres devinaient. J'ai eu le sentiment d'avoir appris bcp de vocabulaire avec les émotions. Par exemple, la différence entre fourbe et malicieux. » (Grayson, étudiant américain); « Je pense que j’ai amélioré un peu parce que j'ai finalement plein de choses à dire pendant le jeu » $(\mathrm{Yu}-$ Ching, étudiante chinoise)

4. La « case vide» est certainement la plus remplie! Parmi les éléments extérieurs à l'innovation mais que l'on ne peut ignorer, surgissent de nombreux éléments personnels, parmi lesquels :

28 - des éléments intimes liés à l'individu : «En ce moment, je me sens désolée pour mes profs, mes parents et moi-même car je ne suis pas faire autant mieux que j'imagine.» (Chengrong, étudiante chinoise); « Je ne sais pas pourquoi je suis devenue un chien ...en fait, quand j'étais petite...souvenir d'enfance» (Shyun, étudiante chinoise); « Normalement, je ne veux pas parler ni en cours ni en public mais au fond de mon cœur, il y a une folle qui attend d'être libéré et je crois que tout le monde l'a vu. » (Yu- 
Ching, étudiante chinoise); « Ce cours pour moi est très différent. Je n'ai jamais eu de cours comme ça avant, ravi et intéressant (...). Si en future, je peut devenir professeur de français en Chine, je voudrai bien donner un cours comme ça : ce cours me donne trop d'idées sur comment encourager les étudiants à participer» (Yanru, étudiant chinois);

29 - des éléments personnels liés à l'apprenant: «Je ne suis pas créatif.» (Paul, étudiant américain); « Pour moi, le plus intéressant, c'est jouer une petite scène : on peut créer, imaginer, s'adapter, travailler ensemble, organiser la façon qu'on parle et s'amuser. " (Yong H, étudiante chinoise); «La spontanéité est difficile pour moi, j'ai l'habitude de préparer tout dans ma tête » (Mizuki, étudiante japonaise);

30 - des éléments personnels liés au prof : «J'aime vos cheveux courts. » (Yuki, étudiante japonaise); «La prof était drôle. Elle nous a fait beaucoup rire.» (Mingfei, étudiante chinoise); « vous me donnez beaucoup d'énergie » (Yu-Ching, étudiante chinoise);

31 - des éléments personnels liés à la méthodologie appliquée en classe : «Madame le prof a toujours des jeux intéressants. » (Weiling, étudiante chinoise); «J'adore ce cours sur la bataille: on s'est battu avec les autres...hahaha, c'était complètement fou.» (YuChing, étudiante chinoise); "J'aimerais bien si on a plus d'occasions de répéter nos sketchs pendant le semestre parce que je crois qu'on apprends beaucoup quand on répète la même chose plusieurs fois. » (Paul, étudiant américain); "J'aime quand on se dépêche pour aller s'asseoir et c'est le dernier assis qui commence en premier » (Shyun, étudiante chinoise); «À mon avis, l'échauffement était très pratique pour les étudiants : d'un côté, on a bien pu préparer le corps et la voix; de l'autre côté, on est devenu plus dynamique de bien s'intégrer dans le cours.» (Lin Meng, étudiante chinoise);

32 - des éléments personnels liés à la vie de classe : « rire, rire, rire, toujours rire...si la vie était comme le cours d'expression théâtrale » (Yao Shen, étudiante chinoise); « tout le monde a créé un personnage: c'était amusant» (Yu-Ching, étudiante chinoise); « l'atmosphère est superbe » (Yanru, étudiante chinoise);

33 - des éléments personnels liés à l'interculturalité : «les sons des animaux peut varier d'une nationalité à l'autre!» (Yao Shen, étudiante chinoise, «Pour moi, c'était intéressant de voir comment le corps montre les sentiments aussi que le visage, et de plus les variations entre des pays différents. » (Emma, étudiante anglaise).

\section{Conclusion}

34 La pratique innovante, rappelle Cros $(2001,2013)$, trouve ses origines dans une difficulté, une frustration, une motivation, une envie d'action personnelle ou encore une exigence institutionnelle. Dans le cas du cours d'expression théâtrale, c'est l'insatisfaction liée à la mise en œuvre de la compétence de production orale qui a généré une novation.

Devenu routinier pour l'enseignant et institutionnalisé pour les membres de l'institution, ce cours a, au fil des années, perdu son côté novateur. Seule la réflexivité sur la pratique continue à engendrer de nouvelles intentions et de nouveaux projets. Grâce à l'écoute (d'abord visuelle) des forces et des faiblesses de cette pratique en classe, notamment à travers la communication non verbale (froncement de sourcils, rires, sourires, plissement de front, etc.), et à la lecture des carnets de bord des 
apprenants, l'innovateur apprend à réinterroger sa pratique. Cela peut concerner la cohérence de sa méthodologie, la pertinence des activités choisies, la prise en compte de l'individu, etc. En l'absence de ces indices, l'innovateur ne pourrait progresser. Fautil encore qu'il en ait envie! Car, rappelle Bodin (2002), l'évaluation, est d'abord affaire de posture, de disposition à remettre en cause les démarches entreprises, à être ouvert à l'inattendu, voire à ce que l'on aurait préféré ne pas voir.

Dans notre cheminement pédagogique, l'évaluation est un processus qui consiste à recueillir des informations pertinentes, à examiner le degré d'inadéquation entre des intentions d'objectifs déterminées au préalable et des réalisations, tout en donnant du sens et/ou redonnant du sens à l'enseignement-apprentissage. C'est l'intuition, l'écoute du vécu de l'expérience et l'action en direct qui servent l'évaluation avec la mise en œuvre de changements progressifs (implication des apprenants dans l'évaluation, mise en place d'un cours partagé, changement de grilles d'évaluation, etc.). Les carnets de bord, au-delà de la légitimité qu'ils donnent à ce cours, témoignent de la métamorphose ou des métamorphoses qui s'opèrent chez l'apprenant. C'est ce processus de transformation qu'il faudrait aujourd'hui analyser pour innover demain : l'apprenant à devenu autre, à partir de lui-même.

En définitive, c'est grâce à une évaluation partagée (l'institution, les membres de l'équipe, les collègues-enseignants, les apprenants, l'innovateur) et ce, dans un continuum infini, que l'innovation ne cesse d'exister. C'est peut-être moins le chemin parcouru que le chemin à parcourir qui donne à l'innovation, un avenir certain.

\section{BIBLIOGRAPHY}

Alix, C., Lagorgette, D. et Rollinat-Levasseur, E. M. (2013). Didactique du Français Langue Étrangère par la Pratique Théâtrale. Chambéry, France : Université de Savoie.

Baldwin, J. et Mederos Syssoeva, K. (1999). La biomécanique de Meyerhold et l'acteur contemporain : comment former l'acteur complet. L'Annuaire théâtral : Revue québécoise d'études théâtrales, (25), 134-150. Repéré à https://doi.org/10.7202/041382ar

Becker, H. S. (2006). Sur le concept d'engagement. SociologieS. Repéré à https:// journals.openedition.org/sociologies/642

Bédard, D. et Béchard, J.-P. (2009). Innover dans l'enseignement supérieur. Dans D. Bédard et J.-P. Béchard (dir.), L'innovation pédagogique dans le supérieur : un vaste chantier (p. 29-43). Paris, France : Presses Universitaires de France. Repéré à https://www.cairn.info/innover-dans-1enseignement-superieur--9782130571476.htm

Bodin, A. (2002). L'évaluation, pratique scientifique et sociale : cas de l'évaluation des pratiques de changement. Dans D. Alexandre (dir.), Évaluer les pratiques innovantes (p. 25-35). Repéré à http://ww2.ac-poitiers.fr/cardie/IMG/pdf/evaluer_pratiques.pdf

Caré, J. M. (1983). Jeux de rôles ou drôles de jeux. Français dans le monde, (176), 75-78. 
Château, A. et Zumbihl, H. (2010). Le carnet de bord, un outil permettant le cheminement vers l'autonomisation dans un dispositif d'apprentissage de l'anglais en ligne ? Alsic, 13. Repéré à https://journals.openedition.org/alsic/1392

Cocton, M.-N. (2007). L'improvisation contextualisée en classe de français langue étrangère : le cas d'apprenants japonais (Thèse de doctorat inédite). Université de Nantes, France.

Cocton, M.-N. (2015). Impliquer les apprenants de FLE dans l'évaluation et l'auto-évaluation en cours d'expression théâtrale. Lidil, (52), 107-127. Repéré à https://doi.org/10.4000/lidil.3851

Conseil de l'Europe (2005). Cadre européen commun de référence - Livre + DVD (Les travaux du conseil de l'Europe) (French Edition). Paris, France : DIDIER.

Cros, F. (1999). L'innovation en éducation et en formation dans tous ses sens. Recherche \& Formation, 31(1), 127-136. Repéré à https://doi.org/10.3406/refor.1999.1576

Cros, F. (2001). Pour une réflexion critique de l'évaluation des innovations scolaires. Les dossiers des Sciences de l'Éducation, 6, 69-79. Repéré à https://doi.org/10.3406/dsedu.2001.963

Cros, F. (2013). L'innovation en formation. Dans A. Jorro (dir.), Dictionnaire des concepts de la professionnalisation (p. 169-175). Louvain-la-Neuve, Belgique : De Boeck Supérieur. doi:10.3917/ dbu.devel.2013.02.0169

Cros, F. (2017). Innovation et société, le cas de l'école. Londres, Royaume Uni : ISTE Editions.

De Ketele, J.-M. (2002). L'évaluation de et dans l'innovation. Dans Bureau de la valorisation des innovations pédagogiques (dir.), Évaluer les pratiques innovantes : actes des journées d'étude des 26 et 27 mars 2001, Saline royal d'Arc-et-Senans, Académie de Besançon (p. 35-42). Paris, France : Centre national de documentation pédagogique. Repéré à http://ww2.ac-poitiers.fr/cardie/IMG/pdf/ evaluer_pratiques.pdf

De Ketele, J.-M. (2010). L'innovation pédagogique dans l'enseignement supérieur : des chemins de traverse aux avenues institutionnelles. Revista portuguesa de pedagogia, 7-24. Repéré à http:// hdl.handle.net/10316.2/5342

Deum, M. (2004). Développer la pratique réflexive et amorcer la professionnalisation en formation initiale des enseignants au travers de la pratique du journal de bord : Analyse d'un dispositif belge de formation. Dans Actes du colloque de l'AIRDF. Québec, Canada : Université Laval. Repéré à http://hdl.handle.net/2268/71444

Huver, E. et Springer, C. (2011). L'évaluation en langues. Paris, France : Didier.

Jorro, A. (2002). De la dynamique de l'action à son évaluation. Dans Bureau de la valorisation des innovations pédagogiques (dir.), Évaluer les pratiques innovantes : actes des journées d'étude des 26 et 27 mars 2001, Saline royal d'Arc-et-Senans, Académie de Besançon (p. 19-25). Paris, France : Centre national de documentation pédagogique. Repéré à http://ww2.ac-poitiers.fr/cardie/IMG/pdf/ evaluer_pratiques.pdf

Le Boterf, G. (2002). De quel concept de compétence avons-nous besoin ? Soins Cadres, (41). Repéré à http://www.guyleboterf-conseil.com/images/Soins\%20cadres.PDF

Lecoq, J. (1997). Le corps poétique : Un enseignement de la création théâtrale. Paris, France : Actes sud. Morin, E. (2015). Penser global. Paris, France : Robert Laffont.

Morlaix, S. (2015). Les compétences sociales à l'école primaire : essai de mesure et effets sur la réussite. Carrefours de l'éducation, 40(2), 183-200. doi:10.3917/cdle.040.0183 
Payet, A. (2010). Activités théâtrales en classe de langue. Paris, France : French and European Publications Inc.

Pierra, G. (2006). Le corps, la voix, le texte : Arts du langage en langue étrangère. Paris, France : L'Harmattan.

Pierré, M. et Treffandier, F. (2012). Jeux de théâtre. Grenoble, France : PUG.

Rouby, E. et Thomas, C. (2014). La construction de compétences collectives en environnement complexe : une analyse en termes d'attention organisationnelle. @GRH, 12(3), 39-74. https:// doi.org/10.3917/grh.012.0039

Ryngaert, J.-P. (1977). Le jeu dramatique en milieu scolaire. Paris, France : TNT Cedic.

Tabensky, A. (1997). Spontanéité et interaction : Le jeu de rôle dans l'enseignement des langues étrangères. Paris, France : L'Harmattan.

Vielmas, M. (1990). À haute voix. Paris, France : Clé international.

Winkin, Y. (1981). La nouvelle communication. Paris, France : Seuil.

\section{NOTES}

1. FLE, https://www.uco.fr/fr/formations/humanites/master-francais-langue-etrangere-fle

2. Ce cours optionnel est proposé aux étudiants étrangers qui viennent apprendre le français langue étrangère en immersion. Il est proposé dans les niveaux B1, B2, C1, à raison de $3 \mathrm{~h}$ par semaine, soit $42 \mathrm{~h}$ par semestre.

3. DELF, diplôme d'études en langue française, est une certification délivrée par le ministère français de l'Éducation nationale.

4. Destinés à des classes entières, ces ateliers dessinent un parcours pédagogique écrit à deux voix (celle d'un professionnel du théâtre et celle d'un enseignant) pour faire pratiquer un art de la scène en classe.

5. Nous avons filmé des apprenants japonais (niveau de langue B1) pendant dix séances étalées sur une année universitaire. Dans le cadre d'une thèse de doctorat (Cocton, 2007), nous avons ensuite analysé ces films en utilisant la grille de Tabensky (1997) et avons proposé des grilles d'évaluation à tester.

6. LIDIL, Cocton (2015).

7. «Implication » et « engagement " n'étant pas synonymes, comme indiqué par Winkin (1981, p. 270) : «Être impliqué dans une activité de circonstance signifie y maintenir une certaine attention intellectuelle et affective, une certaine mobilisation de ses ressources psychologiques; en un mot, cela signifie s'y engager (to be involved in it). [...] Le terme involved (engagé, impliqué) comporte également d'autres connotations dans la langue de tous les jours : celle de commitment

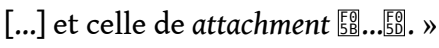

Pour aller plus loin dans la différenciation, on peut également renvoyer à Becker (2006).

8. Nous choisissons de ne pas détailler ce «questionnaire de satisfaction de cours" dans cet article car aucun critère ne correspond à l'innovation.

9. Il n'avait pas été décidé, au départ, que ce carnet de bord servirait à mettre en évidence les cinq critères cités ci-après. Nous avons donc utilisé la technique de l'analyse thématique pour relever des termes redondants correspondant aux critères 1., 2. et 4 . Cette recherche est avant tout descriptive et qualitative.

10. Tous les commentaires des étudiants sont laissés en l'état. Aucune correction orthographique ou syntaxique n'a été effectuée. 
11. La soirée internationale est une soirée, à la fin du semestre, au cours de laquelle les étudiants étrangers sont invités à partager un morceau de leur culture via une chanson, une musique, un diaporama, etc. Le cours d'expression théâtrale peut, si les étudiants sont motivés, proposer une courte séquence reflétant ce qui a été fait en cours.

12. La métamorphose est entendue ici comme transformation sans rupture totale avec le passé (Morin, 2015, p. 83).

\section{ABSTRACTS}

en

This article presents itself as a reflective essay on the evaluation of an innovative activity in French as a Foreign Language (FFL) class: the theatrical expression. Its objective is to question the concept of pedagogical innovation, considered as a new process, which has to be identified and analyzed in order to be made perceptible to the actors of teaching-learning. The first step is to understand the innovative dimension of this teaching-learning practice, both in its content and in its evaluation, before reporting, in a second step, on the metamorphoses described by the learners in their notebook. These transformations, which are linked with the content of the course, the activities, the learner in its relation to learning, the other and the self, play a role in nourishing and fostering reflection on the evaluation of pedagogical evaluation and on the actions to be taken in order to generate a new dynamic in the teaching and learning process.

fr

Cet article se présente comme un essai réflexif autour de l'évaluation d'une activité innovante en classe de Français langue étrangère (FLE) : l'expression théâtrale. Son objectif est d'interroger le concept d'innovation pédagogique, considérée comme un processus inédit, qui, pour être rendu perceptible aux acteurs de l'enseignement-apprentissage, doit être identifié et analysé. Il s'agit, dans un premier temps, de comprendre la dimension novatrice de cette pratique d'enseignement-apprentissage, tant dans son contenu que dans son évaluation, avant de rendre compte, dans un second temps, des métamorphoses décrites par les apprenants dans l'outil nommé carnet de bord. Ces transformations liées au contenu du cours, aux activités, à l'apprenant dans son rapport à l'apprentissage, à l'autre et à soi viennent nourrir la réflexion sur l'évaluation de l'innovation pédagogique, et sur les actions à mettre en œuvre pour générer une nouvelle dynamique dans le processus d'enseignement-apprentissage.

\section{INDEX}

Mots-clés: innovation, métamorphoses, évaluation, apprentissage

\section{AUTHOR}

\section{MARIE-NOËLLE COCTON}

LICIA et CIRPaLL, Université catholique de l'Ouest à Angers, Angers, France, mncocton@uco.fr 